Vietnam Journal of Mechanics, NCST of Vietnam Vol. 21, 1999, No 4 (231 - 238)

\title{
ON UNIQUENESS OF A CLASSICAL SOLUTION OF THE SYSTEM OF NON-LINEAR 1-D SAINT VENANT EQUATIONS
}

\author{
HOANG VAN LAI \\ Institute of Information Technology \\ LE MAU LONG \\ Branch of Institute of Information Technology in HoChiMinh City \\ MAI DINH TRUNG \\ Institute of Mechanics
}

\begin{abstract}
In this paper the theorem of uniqueness of a classical solution of the system of non-linear 1-D Saint Venant equations is proved. This uniqueness theorem is setup for the system of non-linear 1-D Saint Venant equations in canonical form under respective initial and boundary conditions.
\end{abstract}

\section{Introduction}

The system of 1-D Saint Venant equations describes flows in a river or open channel. It became kernel of a mathematical modeling for the river flow simulation. Problem of uniqueness of a classical solution of this system of equations is important especially in the non-linear case.

2. Boundary Condition for the System of 1-D Saint Venant Equations

\subsection{System of 1-D Saint Venant Equations}

The System of 1-D Saint Venant Equations [1] describes a flow in river or open channel system. There are several forms of this system (see [2]). In this paper, we use the system of 1-D Saint Venant equations in the following form:

$$
\begin{array}{ll}
\frac{\partial h}{\partial t}+u \frac{\partial h}{\partial x}+\frac{A}{b} \frac{\partial u}{\partial x}=0 & \text { with } 0 \leq x \leq L \text { and } 0 \leq t \leq T \\
\frac{\partial u}{\partial t}+g \frac{\partial h}{\partial x}+u \frac{\partial u}{\partial x}=-g\left(S_{f}-S_{0}\right) &
\end{array}
$$

where: $\quad h$ - Depth of water in the river/channel 
$u$ - Velocity of the river/channel flow

$A$ - Cross section area of the river/channel flow

$b$ - Width of water in the river/channel

$S_{f}$ - Force due to bottom friction

$S_{0}$ - Force due to gravity

The system of equations (2.1) is devised from the system of equations (2.29) in [2] assuming that cross section $A$ changes slowly along $x$ direction (thus $\left(\frac{\partial A}{\partial x}\right)_{h=\text { const }} \approx$ $0)$.

\subsection{Canonical Form of the System of 1-D Saint Venant Equations}

The system (2.1) is a system of first order partial differential equations. In order to analyze its qualities, it should be re-written in a canonical form (see [3]). The method for transforming a system of linear equations into a canonical form was presented in detail in [3]. Unfortunately, that technique is not applicable for the Saint Venant system of equations (2.1) due to its non-linearity. However (2.1) can be transformed into a canonical form by the following steps:

Step 1. Transforming the system of equations (2.1) into symmetrical form: For this purpose, we present (2.1) in the following vector form:

$$
\frac{\partial}{\partial t} \mathbf{U}+A \frac{\partial}{\partial x} \mathbf{U}=\mathbf{f}
$$

where

$$
\mathbf{U}=\left[\begin{array}{l}
h \\
u
\end{array}\right], \quad A=A(\mathbf{U})=\left[\begin{array}{ll}
u & \frac{A}{b} \\
g & u
\end{array}\right], \quad \mathbf{f}=\left[\begin{array}{c}
0 \\
-g\left(S_{f}-S_{0}\right)
\end{array}\right]
$$

Here matrix $\mathbf{A}$ is not symmetrical. So, to make it symmetrical we need the following transform function:

$$
z=\varphi(h)
$$

where $\varphi(h)$ need to be defined so that matrix $B$ derived from (2.2) is a symmetrical matrix. It could be proved that $\varphi(h)$ should have a form of:

$$
\varphi(h)=\sqrt{g} \int_{0}^{h} \sqrt{\frac{b(\tau)}{A(\tau)}} d \tau \text { so } \varphi^{\prime}(h)=\sqrt{g} \sqrt{\frac{b(h)}{A(h)}} .
$$

From (2.4) we have $\varphi^{\prime}(h)>0$ so $\varphi(h)$ is a monotone increasing function. Therefore exists an inverse function $h=\varphi^{-1}(z)$. We shall suppose that the function $h=\varphi^{-1}(z)$ is smooth. 
Next, note that

$$
\varphi^{\prime}(h) \cdot \frac{A(h)}{b(h)}=\frac{g}{\varphi^{\prime}(h)}=\sqrt{\frac{g A(h)}{b(h)}} \equiv c
$$

so the system (2.2) can be re-written in'the following form:

$$
\frac{\partial}{\partial t} \mathbf{U}^{\prime}+B \frac{\partial}{\partial x} \mathbf{U}^{\prime}=\mathbf{f}^{\prime}
$$

here:

$$
\mathbf{U}^{\prime}=\left[\begin{array}{l}
z \\
u
\end{array}\right], \quad B=B\left(\mathbf{U}^{\prime}\right)=\left[\begin{array}{ll}
u & c \\
c & u
\end{array}\right], \quad \mathbf{f}^{\prime}=\left[\begin{array}{c}
0 \\
-g\left(S_{f}-S_{0}\right)
\end{array}\right]
$$

It is easy to find that eigenvalues for the matrix $B$ are:

$$
\lambda_{1}=u+c \text { and } \lambda_{2}=u-c
$$

Lemma 1. The eigenvectors of the matrix $B$ are:

$$
\left[\begin{array}{l}
1 \\
1
\end{array}\right] \text { and }\left[\begin{array}{c}
1 \\
-1
\end{array}\right]
$$

The proof of this lemma is simple.

Step 2. We have the following matrix of the eigenvectors of the matrix B:

$$
E=\left[\begin{array}{cc}
1 & 1 \\
1 & -1
\end{array}\right] \text { so } E^{-1}=\frac{1}{2}\left[\begin{array}{cc}
1 & 1 \\
1 & -1
\end{array}\right]
$$

Since the matrix $E$ (and consequently $E^{-1}$ ) is not depend on $z$ and $u$. Thus we can transform system (2.2) into the following canonical form:

$$
\begin{aligned}
& \frac{\partial w_{1}}{\partial t}+\lambda_{1}(\mathbf{w}) \frac{\partial w_{1}}{\partial x}=\psi(\mathbf{w}) \\
& \frac{\partial w_{2}}{\partial t}+\lambda_{2}(\mathbf{w}) \frac{\partial w_{2}}{\partial x}=-\psi(\mathbf{w})
\end{aligned}
$$

with

$$
\begin{aligned}
w_{1} & =\frac{\varphi(h)+u}{2} & w_{2} & =\frac{\varphi(h)-u}{2} \\
\lambda_{1} & =u+c & \lambda_{2} & =u-c \\
\mathbf{w} & =\left(w_{1}, w_{2}\right) & \psi(\mathbf{w}) & =g\left(S_{f}-S_{0}\right) / 2
\end{aligned}
$$


The form of (2.5) is suitable for qualitative investigation of the system of Saint Venant equations (2.1). We will use this form in proving the uniqueness of a classical solution of the system (2.1). In several other papers, the system of equations (2.1) is written in the following characteric form:

$\frac{\partial Q}{\partial t}+(u \pm c) \frac{\partial Q}{\partial x}+B(-u \pm c)\left[\frac{\partial y}{\partial t}+(-u \pm c) \frac{\partial y}{\partial x}\right]=\left[i b+\left(\frac{\partial A}{\partial x}\right)_{h=\text { const }}\right] u^{2}-\frac{g A Q|Q|}{k^{2}}$

where: $y$ - water level; $y=h+y_{b} ; y_{b}$ - bottom tevel. Obviously, the form (2.5) is simpler than the form (2.6). In the next sections, some time, instead of $w_{1}, w_{2}$, we shall use $h$ and $u$. It is easy to see that

$$
h=\varphi^{-1}\left(w_{1}+w_{2}\right) \quad \text { and } \quad u=w_{1}-w_{2}
$$

\subsection{Boundary Condition for the System of Equations (2.5)}

For the system of Saint Venant equations in form (2.5) we need initial conditions as:

$$
w_{1}(x, 0)=w_{1}^{I}(x) \quad w_{2}(x, 0)=w_{2}^{I}(x) \quad \text { while } 0 \leq x \leq L
$$

The boundary conditions for system (2.5) need to be chosen in dependence of the signs of $\lambda_{1}$ and $\lambda_{2}$ at the boundaries. In more details they are :

a) At $x=0$ : If

$\lambda_{1}<0 ; \lambda_{2}<0$ then no boundary conditions are needed

$\lambda_{1}>0 ; \lambda_{2}<0$ then one boundary condition

$$
w_{1}(0, t)=w_{1}^{L}(t) \text { is needed }
$$

$\lambda_{1}>0 ; \lambda_{2}>0$ then two boundary conditions

$$
w_{1}(0, t)=w_{1}^{L}(t) \text { and } w_{2}(0, t)=w_{2}^{L}(t) \text { are needed }
$$

b) And at $x=L:$ If

$$
\begin{aligned}
& \lambda_{1}<0 ; \lambda_{2}<0 \text { then two boundary conditions } \\
& \qquad w_{1}(L, t)=w_{1}^{p}(t) \text { and } w_{2}(L, t)=w_{2}^{p}(t) \text { are needed }
\end{aligned}
$$

$\lambda_{1}>0 ; \lambda_{2}<0$ then one boundary condition

$$
w_{2}(L, t)=w_{2}^{p}(t) \text { is needed }
$$

$\lambda_{1}>0 ; \lambda_{2}>0$ then no boundary conditions are needed

The boundary conditions (2.8.1)-(2.8.6) are called in common as boundary condition (2.8). 


\section{Uniqueness Theorem}

\subsection{Condition on Bottom Resistance for the Uniqueness Theorem}

We consider function $S_{f}$ in the form of: (see [2], formula (2.39))

$$
S_{f}=\alpha(h) \beta(u) \quad \alpha(h)=\frac{g}{C^{2} R} \quad \beta(u)=|u| u
$$

with $R=R(h)$ - Hydraulic radius; $C$ - the Chezy coefficient

The function $S_{0}$ was defined as in [2]:

$$
S_{0}=-\frac{\partial y_{b}}{\partial x} \quad \text { with } y_{b}-\text { bottom level. }
$$

We consider only case when $R(h)>0$.

\subsection{Uniqueness Theorem}

Assuming that in a closed area $\bar{\Omega}=[0, L] \times[0, T]$ there exists a classical solution $\mathbf{w}(x, t)=\left[w_{1}(x, t), w_{2}(x, t)\right]$ of the boundary problem for the system of Saint Venant equations (2.5), under the initial conditions (2.7) and the boundary conditions (2.8). Then this solution is unique.

\subsection{Proof of the Uniqueness Theorem}

The tool used for proving the Theorem 3.2 will be integrals of energy (see [3]). Due to non-linearity of the system of Saint Venant equations, the proof of the uniqueness theorem in this case is more complicated in comparison with the hyperbolic linear case (see [3]).

We will prove the unique solution in classical means, i.e.

1) The functions of $w_{1}, w_{2}$ belongs to the $C^{(1)}(\Omega)$.

2) The functions of $\mathbf{w}_{1}, \mathbf{w}_{2}$ satisfy system of equations (2.5), initial conditions (2.7) and boundary conditions (2.8).

We will prove the Theorem 3.2 by contrarious method: Suppose that there exist two classical solutions of the system (2.5) as $\mathbf{w}^{\prime}$ and $\mathrm{w}^{\prime \prime}$ satisfied the initial conditions (2.7) and the boundary conditions (2.8):

$$
\begin{aligned}
\mathbf{w}^{\prime} & =\mathbf{w}^{\prime}(x, t)=\left[w_{1}^{\prime}(x, t), w_{2}^{\prime}(x, t)\right] \\
\mathbf{w}^{\prime \prime} & =\mathbf{w}^{\prime \prime}(x, t)=\left[w_{1}^{\prime \prime}(x, t), w_{2}^{\prime \prime}(x, t)\right]
\end{aligned}
$$

Define $\mathbf{w}=\mathbf{w}^{\prime}-\mathbf{w}^{\prime \prime}$, and here in contrary with the linear case we can not conclude that $\mathbf{w}$ satisfies the system of Saint Venant equations. However we can evaluate the integrals of energy for $\mathbf{w}$. Indeed since $w_{1}^{\prime}(x, t), w_{2}^{\prime}(x, t), w_{1}^{\prime \prime}(x, t), w_{2}^{\prime \prime}(x, t)$ belong to the $C^{(1)}(\Omega)$ then there exists a constant $\alpha, 0 \leq \alpha<\infty$ so that 


$$
\begin{aligned}
\left|w_{k}^{\prime}\right| & \leq \alpha \text { and }\left|w_{k}^{\prime \prime}\right| \leq \alpha \\
\left|\frac{\partial}{\partial x} w_{k}^{\prime}\right| \leq \alpha & \text { and }\left|\frac{\partial}{\partial x} w_{k}^{\prime \prime}\right| \leq \alpha \quad \text { with } \quad k=1,2
\end{aligned}
$$

Thus

$$
\left|w_{k}\right| \leq 2 \alpha \text { and }\left|\frac{\partial}{\partial x} w_{k}\right| \leq 2 \alpha \text { with } k=1,2
$$

Since both $w^{\prime}$ and $w^{\prime \prime}$ satisfy system of equations (2.5) then we have

$$
\frac{\partial w_{1}}{\partial t}+\lambda_{1}\left(\mathbf{w}^{\prime}\right) \frac{\partial w_{1}^{\prime}}{\partial x}-\lambda_{1}\left(\mathbf{w}^{\prime \prime}\right) \frac{\partial w_{1}^{\prime \prime}}{\partial x}=-\left[\psi\left(\mathbf{w}^{\prime}\right)-\psi\left(\mathbf{w}^{\prime \prime}\right)\right]
$$

Subtracting and adding to the left hand side of (3.4) the term $\lambda_{1}\left(w^{\prime}\right) \frac{\partial w_{1}^{\prime \prime}}{\partial x}$, then we have

$$
\frac{\partial w_{1}}{\partial t}+\lambda_{1}\left(\mathbf{w}^{\prime}\right) \frac{\partial w_{1}}{\partial x}+\left[\lambda_{1}\left(\mathbf{w}^{\prime}\right)-\lambda_{1}\left(\mathbf{w}^{\prime \prime}\right)\right] \frac{\partial w_{1}^{\prime \prime}}{\partial x}=-S_{1}
$$

where $S_{1} \equiv \psi\left(\mathbf{w}^{\prime}\right)-\psi\left(\mathbf{w}^{\prime \prime}\right)$

Similarly, we also have

$$
\frac{\partial w_{2}}{\partial t}+\lambda_{2}\left(\mathbf{w}^{\prime}\right) \frac{\partial w_{2}}{\partial x}+\left[\lambda_{2}\left(\mathbf{w}^{\prime}\right)-\lambda_{2}\left(\mathbf{w}^{\prime \prime}\right)\right] \frac{\partial w_{2}^{\prime \prime}}{\partial x}=S_{1}
$$

Multiply (3.5) with $2 w_{1}$ and (3.6) with $2 w_{2}$ then sum up them together we get that

$$
\frac{\partial}{\partial t}\left(w_{1}^{2}+w_{2}^{2}\right)+\frac{\partial}{\partial x}\left[\lambda_{1}\left(\mathbf{w}^{\prime}\right) w_{1}^{2}+\lambda_{2}\left(\mathbf{w}^{\prime}\right) w_{2}^{2}\right]=S_{2}-2 S_{1}\left(w_{1}-w_{2}\right),
$$

where

$$
\begin{aligned}
S_{2}= & w_{1}^{2}\left[\frac{\partial}{\partial x} \lambda_{1}\left(\mathbf{w}^{\prime}\right)\right]+w_{2}^{2}\left[\frac{\partial}{\partial x} \lambda_{2}\left(\mathbf{w}^{\prime}\right)\right]- \\
& -2 w_{1}\left(\lambda_{1}\left(\mathbf{w}^{\prime}\right)-\lambda_{1}\left(\mathbf{w}^{\prime \prime}\right)\right) \frac{\partial w_{1}^{\prime \prime}}{\partial x}-2 w_{2}\left(\lambda_{2}\left(\mathbf{w}^{\prime}\right)-\lambda_{2}\left(\mathbf{w}^{\prime \prime}\right)\right) \frac{\partial w_{2}^{\prime \prime}}{\partial x} .
\end{aligned}
$$

Consider the energy integral as follows:

$$
I(t)=\int_{0}^{L}\left(w_{1}^{2}+w_{2}^{2}\right) d x .
$$

Integrating (3.7) with respect to $x$ from 0 to $L$ and with respect to $t$ from $t_{1}$ to $t_{2}$ we can get: 


$$
I\left(t_{2}\right)-I\left(t_{1}\right)+J=\int_{t_{1}}^{t_{2}} \int_{0}^{L}\left[S_{2}-2 S_{1}\left(w_{1}-w_{2}\right)\right] d x d t
$$

where

$$
J=\int_{t_{1}}^{t_{2}}\left\{\left.\left[\lambda_{1}\left(w^{\prime}\right) w_{1}^{2}+\lambda_{2}\left(w^{\prime}\right) w_{2}^{2}\right]\right|_{x=L}-\left.\left[\lambda_{1}\left(w^{\prime}\right) w_{1}^{2}+\lambda_{2}\left(w^{\prime}\right) w_{2}^{2}\right]\right|_{x=0}\right\} d t
$$

Due to boundary condition (2.8) we have $J \geq 0$ : Indeed since $\left(w_{1}\right)^{2} \geq 0$ and $\left(w_{2}\right)^{2} \geq 0$, then at the boundary $x=0$, if $\lambda_{1}<0$ (i.e. $-\lambda_{1}>0$ ) then $-\lambda_{1}\left(w^{\prime}\right) w_{1}^{2} \geq$ 0 , if $\lambda_{1}>0$ (i.e. $-\lambda_{1}<0$ ) then the identical boundary condition for $w^{\prime}$ and $w^{\prime \prime}$ (thus $w_{1}^{\prime}$ and $w_{1}^{\prime \prime}$ ) makes $\left(w_{1}\right)^{2}=0$ since $w_{1}=w_{1}^{\prime}-w_{1}^{\prime \prime}$. Similarly for $\lambda_{1}$ and boundary $x=L$ and for the other eigenvalue $\lambda_{2}$.

According to the technique applied in [2] now we have to estimate the right hand side of (3.9) via $I(t)$.

After long computation, we can get the following results:

\section{Lemma 2.}

$$
\begin{aligned}
& \left|S_{2}\right| \leq \alpha_{1}\left[\left(w_{1}\right)^{2}+\left(w_{2}\right)^{2}\right] \\
& \left|2 S_{1}\left(w_{1}-w_{2}\right)\right| \leq \alpha_{2}\left[\left(w_{1}\right)^{2}+\left(w_{2}\right)^{2}\right],
\end{aligned}
$$

where $\alpha_{1}$ and $\alpha_{2}$ are positive constants which depend only on $\alpha$ in (3.1) - (3.3).

From (3.9) we have the following equality

$$
I\left(t_{2}\right)+J=I\left(t_{1}\right)+\int_{t_{1}}^{t_{2}} \int_{0}^{t}\left[S_{2}-2 S_{1}\left(w_{1}-w_{2}\right)\right] d x d t
$$

and the from (3.10), (3.11) and the fact that $J \geq 0$ we obtain the inequality

$$
I\left(t_{2}\right) \leq I\left(t_{1}\right)+\left(\alpha_{1}+\alpha_{2}\right) \int_{t_{1}}^{t_{2}} I(t) d t
$$

We shall use the following lemma of integral inequality (see [3], p. 123).

\section{Lemma 3 (integral inequality)}

Suppose that with $t, 0 \leq t \leq T$, a continuous function $I(t), I(t) \geq 0$, has derivative and for every $t_{1}, t_{2}, 0 \leq t_{1} \leq t_{2} \leq T$, we have following inequality: 


$$
I\left(t_{2}\right) \leq I\left(t_{1}\right)+M \int_{t_{1}}^{t_{2}} I(t) d t+N \int_{t_{1}}^{t_{2}} \sqrt{I(t)} d t \quad \text { with } \quad M>0, N \geq 0
$$

then:

$$
\sqrt{I(t)} \leq \sqrt{I(0)} e^{M t / 2}+\frac{N}{M}\left(e^{M t / 2}-1\right)
$$

From (3.12) we have

$$
I(t) \leq I(0) \exp \left[\left(\alpha_{1}+\alpha_{2}\right) t\right]
$$

Because both solutions $w^{\prime}, w^{\prime \prime}$ satisfied the initial condition $(2.7)$, then $I(0)=0$. From the inequality (3.13) we get that $I(t)=0$, i.e. $w_{1}^{\prime}=w_{1}^{\prime \prime}$ and $w_{2}^{\prime}=w_{2}^{\prime \prime}$. The Theorem 3.2 is proved.

\section{Conclusion}

In this paper, the uniqueness of the classical solution of the system of 1-D Saint Venant equations in the canonical form (2.5) is proved. It is important to get some analogical facts for the system of 1-D Saint Venant equations in the "natural" form (2.1). These questions will be discussed in the next papers.

Acnowledgement This publication is completed with financial support from the Research Program on Basic Science. The authors would like to thank Prof. Dr. Nguyen Van Diep and Ngo Huy Can for their useful remarks.

\section{REFERENCES}

1. Saint-Venant (Barre de) 1871 C.R. Acad. Sci. Paris, 73, 148-154, 237-240. Theory of unsteady water flow with application to river floods and to propagation of tides in river channels.

2. Cunge J. A., Holly F. M. and Verwey A. 1980 "Practical Aspects of Computational River Hydraulics", Pitman Advanced Publishing Program, Boston.

3. Gordunov S. K. Equations of Mathematical Physics. Moscow, 1971.

Received May 4, 1999

ĐỊNH LÝ DUY NHẤT NGHIỆM CỔ ĐIỂN CƯA Hệ PHUƠNG TRÌNH SAINT VENANT PHI TUYẾN MộT CHIỀU

Trong bài báo, định lý duy nhất của nghiệm cổ điển của hệ phương trình Saint Venant phi tuyến một chiều đã được chứng minh. Định lý duy nhất được thiết lập cho hệ phương trình ở dạng chính tắc với các điều kiện biên và điều kiện ban đầu tương ứng. 